\title{
Thermodynamics of an Asymmetric DNA Holliday Junction
}

\author{
$\mathrm{Li} \mathrm{Li}$ \\ South China University of Technology, Guangzhou 510640, P. R China. \\ E-mail: li.z.li@hotmail.com
}

doi:10.5618/bio.2011.v1.n1.2 || Received: Jan. 18, 2011, Accepted: Feb. 2, 2011, Available online: Feb. 21, 2011

\begin{abstract}
An asymmetric junction of known crystal structure is chosen to investigate its thermodynamic properties in solutions using nano-DSC. The thermal scans are modeled with two-state functions to derive the van't Hoff thermodynamic qualities of junction unfolding. It is found that junctions of open-X unfold with two sites simultaneously and independently. The junction conformational switch from open-X to stacked-X was detected in a sodium phosphate buffer with ionic strength of $113 \mathrm{mM}$. Furthermore, the hydration of junctions is investigated by applying different DSC chamber pressure. The positive molar volume change indicates that the studied junctions are not intensively hydrated. The hydration of junction cross-over is less intensive than that of junction arms. This study can provide valuable insight into junction migration mechanism and interactions of junctions with other molecules such as ions and proteins.
\end{abstract}

Keywords: Asymmetric DNA junctions; DSC; unfolding enthalpy; open-X and stacked-X; ionic strength; pressure perturbation.

\section{Introduction}

Holliday Junctions are the key intermediate in most recombination processes that are responsible for the genome integrity and diversity. A general structure of Holliday junction in solution has been deduced from the structure of four junctions constructed from related sequence by gel electrophoresis [1]. It was found that junctions adopt an X-like structure involving colinear helix-helix association, and cation binding is essential for the correct formation. In the absence of cations, the helical arms of the junction are not stacked but fully extended in a square configuration to show an open-X form. In presence of divalent ions $\mathrm{Mg}^{2+}$, the helical arms of the junction are stacked to have antiparallel stacked-X (non-crossed) structure. The antiparallel stacked-X structure has two possible isomers with different stacked partners, and the sequence at the junction is critical to the choice of the two isomers.

In order to build bridge between dynamic properties of the junction in solution and its structural properties in crystal, the crystal structure was studied for an asymmetric junction [2] constructed from four unique strands based on the constructed strands for Junction 3 of previous study [1], but having a four base pair arm stacked over a six base pair arms on either side of the junction cross-over, instead of having a six base pair arms stacked over an eight base par arms. The crystal structure is found to be is an antiparallel stacked-X form with semi-continuous helices forming a right-handed rotation angle of $56.5^{\circ}$, as predicted from solution studies [1]. In contrast to symmetric junctions with junction cross-over stabilized by a unique set of sequence-specific interactions at the U-turn region $[3,4]$ $[5,6]$, this asymmetric junction lacks of specific intramolecular interaction at the U-turn region, despite a short distance interaction $(2.8 \AA)$ is observed between the C5-methyl of thymine T) and the phosphate oxygen of the neighbor Cytosine $\mathrm{C}$ at the U-turn of strand D (see anti-parallel stacked- $X$ in Fig. 2).

The thermodynamic quality is another important property of Holliday Junction. Unfolding/ melting of DNA Holliday junctions can provide energetic information to their genetic functions and structures. Differential scanning calorimetry (DSC) is a direct tool to measure the thermodynamic quantities of molecules in their native states in solutions. Many DSC studies have been carried out in biological macromolecules, in particular nucleic acids[7,8], lipids [9], proteins [7, $10,11]$ and their complexes in order to obtain the energetic information of biological molecule's structures and their interactions with partner molecules $[10,11]$.

In this work, the same asymmetric junction from crystal study was chosen to investigate its thermodynamic properties in solutions using nano-DSC. The annealed mixture solutions of four unique DNA sequences were prepared in different buffers for the conformational switch study, and the thermal scans of the solutions were preformed under different DSC chamber pressure to investigate the hydration of junctions. The van't Hoff thermodynamic qualities of junction unfolding were derived from the thermal scans through modeling. The junction conformation and conformational switch was addressed based on the 
determined van't Hoff thermodynamic parameters, together with the junction unfolding mechanism and hydration status of the junctions. The study results provide energetic insights to branch migration and molecular interactions between junctions with proteins, ions and etc.

\section{Materials and methods}

2.1 Preparation of DNA solutions. DNA oligos of four unique sequences 5'-CCGAGTCCTA-3' (A) , 5'CTCAACTCGG-3' (B), 5'-TAGGGGCCGA-3'(C), 5'TCGGCCTGAG-3' (D) were ordered from IDT with dual purification. Mixture(ABCD) were prepared by mixing $50 \mu \mathrm{M}$ of sequences $\mathrm{A}, \mathrm{B}, \mathrm{C}$ and $\mathrm{D}$, respectively, in $50 \mathrm{mM}$ sodium cacodylate buffer $(\mathrm{pH} \mathrm{7.0)}$. The mixed DNA solutions were pre-heated at $90{ }^{\circ} \mathrm{C}$ for 15 minutes and then kept in a heat-proved foam box overnight to cool down slowly to allow the formations of $\mathrm{ABCD}$ junctions. $50 \mu \mathrm{M} \mathrm{ds}(\mathrm{AB})$ duplex solutions in $50 \mathrm{mM}$ sodium cacodylate buffer $(\mathrm{pH} 7.0)$ were prepared in similar way for a comparison experiment to that of ABCD junctions. In order to investigate the melting behaviors of $A B C D$ junctions in presence of different buffers, $50 \mu \mathrm{M}$ mixture(ABCD) solutions were also prepared in 50mM sodium phosphate buffer ( $\mathrm{pH} 7.0)$.

The annealing of all the studied DNA solutions was preformed the day right prior to DSC measurements.

\subsection{Differential Scanning Calorimetry (DSC).}

A TA nano differential scanning calorimeter (TA Inc.) was used for the DSC experiments in this work. A buffer baseline was obtained prior to sample measurements by running a thermal scanning at the identical scanning conditions as those designed for DNA solutions measurements, with both of sample and reference cells filled with the same buffer. The thermal scanning was run from $0{ }^{\circ} \mathrm{C}$ to around $100-120{ }^{\circ} \mathrm{C}$ at a rate of $1{ }^{\circ} \mathrm{C}$ per minute with a 15 minutes' preequilibration time per cycle. 3-4 cycles of scanning were run for each sample. The DSC sample chamber was pressurized to $3 \mathrm{~atm}$ for standard measurements and 5 atm for a pressure perturbation study.

All the data analysis was performed using NanoAanlysis software provided by TA Instrument. The buffer baseline can be automatically subtracted using the software.

The thermal scans were modeled with two-state functions [12] derived from Van't Hoff equations to obtain the van't Hoff's transition enthalpy, $\Delta H^{V H}$, and its associated transition temperature, $T_{m}{ }^{V H}$. The measured thermodynamic quantities by DSC from each solution were obtained by averaging the results from 3 scans on the solutions.

\section{Results and discussion}

3.1 Duplexes (AB) and asymmetric junctions (ABCD) in solutions. The background (sample baseline) correction is always is crucial to the estimation of both calorimetric and van't Hoff enthalpies of a DSC transition since there are no agreements on a systematic solution for thermal scanning background corrections. To prevent raising fake peaks or losing real small peaks in the thermal scanning curves from incorrect background corrections, Fig. 1 shows the thermal scans with buffer baseline subtractions only but no background corrections for the solutions of $50 \mu \mathrm{M}$ AB duplexes and $50 \mu \mathrm{M}$ mixture of $\mathrm{A}, \mathrm{B}, \mathrm{C}$, and $\mathrm{D}$ sequences. The thermal scans are measured from the annealed DNA solutions in $50 \mathrm{mM}$ sodium cacodylate (pH 7.0) buffer. The major peak in the unfolding profile of $\operatorname{ds}(A B)$ is centered around $19^{\circ} \mathrm{C}$, as indicated by the dotted vertical line in Fig.1. There are a broad peaks at higher temperature between $50-90^{\circ} \mathrm{C}$. This broad peak is either from unfolding of some unknown stable DNA structures formed from sequences $\mathrm{A}$ and $\mathrm{B}$ with very low population, or heat capacity change of single strands of $\mathrm{A}$ and $\mathrm{B}$ with temperatures.

In this work there are no evidences to exclude either possibility. Compared with the unfolding profile of ds(AB), Mixture(ABCD)'s unfolding profile also show a small peak around $19^{\circ} \mathrm{C}$, which is hardly perceptible without a direct comparison to that of $\mathrm{ds}(\mathrm{AB})$. This is an important reason to show the thermal scanning curves with no background corrections here. This shoulder peak with the same centered temperature as the unfolding temperature of $\mathrm{ds}(\mathrm{AB})$ indicates that there are some small amount of $\mathrm{ds}(\mathrm{AB})$ duplexes formed in the mixture of $(A B C D)$. The major peak shown in the unfolding profile of mixture (ABCD) with highest midpoint temperature $\left(\sim 80^{\circ} \mathrm{C}\right)$ is believe to the unfolding peak of (ABCD) junctions [Junct(ABCD) for short]. Between this major peak and the shoulder peak, there is another relative strong peak centered at around $35^{\circ} \mathrm{C}$. What DNA conformation(s) does this peak correspond to? To answer this question, the approximate free energy of all the possible duplexes formed from a mixture of DNA sequences A, B, C, D in $50 \mathrm{mM} \mathrm{Na}^{+}$are calculated using IDT's OligoAnalyzer 3.1. The calculated results are shown in Table $\mathbf{1}$ with only the most popular conformations. The duplexes with most negative free energy is ds(CD) with 6 complementary Watson-Crick base pairs, and the duplex ds(AB) with 6 Watson-Crick base pairs has the second most negative free energy. 
$\mathrm{ds}(\mathrm{AB})$ and ds(CD) are two duplexes with most WatsonCrick base pairs. Based on these calculated results, it is easy to predict the peak centered at $35^{\circ} \mathrm{C}$, sitting between the unfolding peaks of $\mathrm{ds}(\mathrm{AB})$ and Junct(ABCD), is belonged to the unfolding from duplexes (CD). The population of $\mathrm{ds}(\mathrm{CD})$ is higher than $\mathrm{ds}(\mathrm{AB})$ based on their calculated free energies, therefore the unfolding peak of $\mathrm{ds}(\mathrm{CD})$ is stronger than that of ds $(A B)$ in unfolding profile of Junct (ABCD) in Fig. 1. In conclusion, in the annealed mixture of $\mathrm{A}, \mathrm{B}, \mathrm{C}$, and $\mathrm{D}$, there are a certain amount of $\mathrm{ds}(\mathrm{CD})$ and a small amount of $\mathrm{ds}(\mathrm{AB})$ besides the major Junct (ABCD) conformations (See Fig. 2). Does Junct (ABCD) adopt an antiparallel stacked-X, a parallel stacked-X or an open-X form? This will be discussed below according to the experimental results.

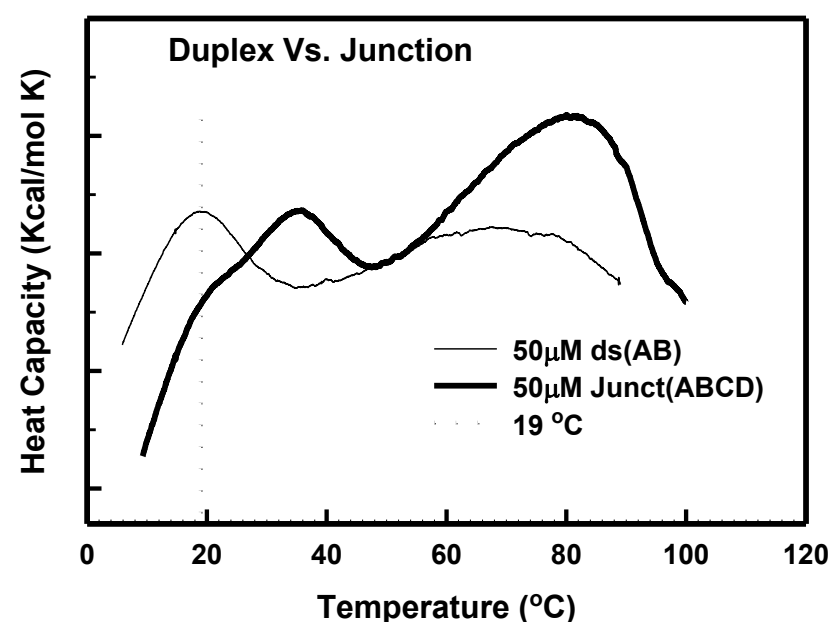

Fig. 1.Excess heat capacity as a function of temperature of $50 \mu \mathrm{M}$ mixture $(\mathrm{ABCD})$ and $50 \mu \mathrm{M}$ duplex (AB), corrected for buffer baseline only but no sample background corrections. The solutions are prepared in $50 \mathrm{mM}$ sodium cacodylate buffer. The dotted vertical line is the reference of $19^{\circ} \mathrm{C}$.

Table 1. The calculated free energies (Kcal/mole) for all the possible duplexes from the mixture of DNA sequences A, B, C, and D. The numbers of complementary Watson-Crick base-pairing for each duplex are also listed. The results are calculated using IDT's OligoAnalyzer 3.1 located on http://www.idtdna.com/analyzer/Applications/OligoAnalyzer

\begin{tabular}{lllll}
\hline $\begin{array}{l}\text { DNA } \\
\text { Sequence }\end{array}$ & A & B & C & D \\
\hline A & $\Delta \mathrm{G}=-3.61$ & $\Delta \mathbf{G}=\mathbf{- 1 1 . 2}$ & $\Delta \mathrm{G}=-5.63$ & $\Delta \mathrm{G}=-8.26$ \\
& Base Pairs: 2 & Base Pairs: 6 & Base Pairs: 4 & Base Pairs: 4 \\
B & -- & $\Delta \mathrm{G}=-3.61$ & $\Delta \mathrm{G}=-8.26$ & $\Delta \mathrm{G}=-5.13$ \\
& & Base Pairs: 2 & Base Pairs: 4 & Base Pairs: 4 \\
C & -- & - & $\Delta \mathrm{G}=-9.28$ & $\Delta \mathbf{G}=\mathbf{- 1 4 . 4 6}$ \\
& & & Base Pairs: 4 & Base Pairs: $\mathbf{6}$ \\
D & -- & -- & $\Delta \mathrm{G}=-9.28$ \\
& & & & Base Pairs: 4 \\
\hline
\end{tabular}

Since the annealed mixture (ABCD) solution of does not form junctions solely, the concentration of junctions cannot be determined, so does the concentrations of bother $\mathrm{ds}(\mathrm{AB})$ and $\mathrm{ds}(\mathrm{CD})$. Without the exact concentrations, the unfolding calorimetric enthalpy of Junct (ABCD) and duplexes cannot be calculated. However the unfolding van't Hoff enthalpy does not affected by the concentrations and is only model-dependent, thus only the van't Hoff enthalpies and their derived thermodynamic qualities are reported in this work. Fig. 3 shows the deconvoluting of the unfolding profile of $\mathrm{ds}(\mathrm{AB})$ formed in $50 \mu \mathrm{M}$ solutions, corrected for background (sample baseline) using a $3^{\text {rd }}$ order polynomial function. The experimental $\mathrm{ds}(\mathrm{AB})$ unfold-ing curve is 
modeled well with a two-model function. This is not surprise since most duplexes are found to unfold in a manner of two states. The two-state modeling results in a van't Hoff unfolding enthalpy $\Delta H^{V H}=42.1 \mathrm{Kcal} / \mathrm{mol}$ and an unfolding temperature $T_{m}{ }^{V H}=18.5{ }^{\circ} \mathrm{C}$ for $\mathrm{AB}$ duplexes. This van't Hoff unfolding enthalpy of $10 \mathrm{mer}$
ds(AB) is around 4 9 $\mathrm{Kcal} / \mathrm{mol}$ lower than those of 8mer DNA duplexes [13]. This may be due to the factor that there are only 6 complementary Watson-Crick basepairings in the 10-mer duplex $\mathrm{AB}$. Due to the same reason, the unfolding temperature of $\operatorname{ds}(\mathrm{AB})$ is much lower than expected.

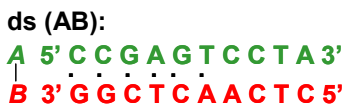

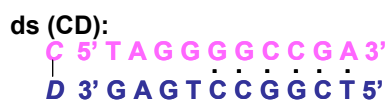

ds (CD): C 5 ; $\mathrm{TAG} \mathrm{G} \mathrm{G} \mathrm{G} \mathrm{C} \mathrm{C} \mathrm{G} \mathrm{A} 3$ D 3' G A G T $\dot{C} \dot{C} \dot{G} \dot{G} \dot{C} \dot{T} 5^{\prime}$

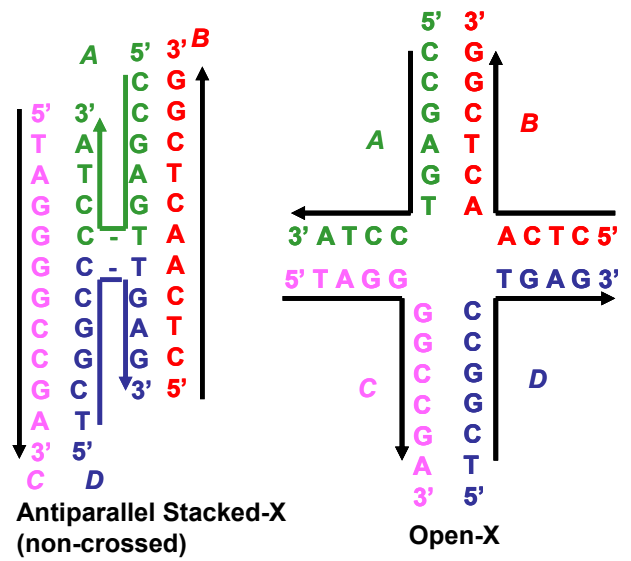

Fig. 2. Schematics of ds $(A B), d s(C D)$ and Junct(ABCD) with three possible conformations: parallel stacked-X, antiparallel stacked-X and open-X .

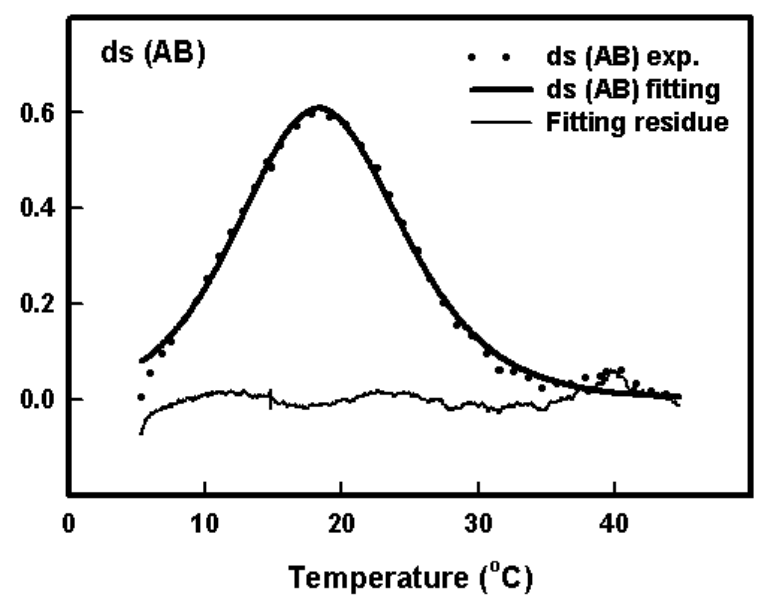

Fig. 3. Deconvolution of the heat capacity scan of ds $(\mathrm{AB})$ from $\sim 50 \mu \mathrm{M}$ ds(AB) solutions to a single component of two-state function.

As discussed above, in the annealed solution of $50 \mu \mathrm{M}$ mixture $(\mathrm{ABCD})$ in $50 \mathrm{mM}$ sodium cacodylate buffer (7.0), there are a certain amount of ds(CD) and a small amount of $\mathrm{ds}(\mathrm{AB})$ besides the major Junct (ABCD) conformations. Fig. 4 shows the unfolding thermal scans of all these DNA conformations in the mixture solution. The experimental curve in heavy dotted curve has been corrected for the sample background. The four lighter solid black curves from left to right are the two- state modeling of unfolding of ds (AB) (1), ds(CD) (2) and Junct (ABCD) (3 and 4), respectively. Different from both of ds $(A B)$ and $d s(C D)$, Junct $(A B C D)$ is found to be modeled with two components of two-state function instead of one single component. The two twostate-function components are overlapping and indicate that unfolding of $\mathrm{ABCD}$ junctions involves two independent cooperative transitions. Both transitions proceed with significant heat absorption and heat 
capacity increment. The sum of all these modelings is plotted in heavy solid curve, which is in good agreement with the experimental data in dotted curve. The studied temperature range does not completely cover the unfolding process of junctions but the unfolding peak can be extended theoretically to a completed point by extending the second modeling component based on its symmetry. The extended portion of the second component is plotted in light red curves in Fig. 4. The same extension is done for the first component of junctions' modelings but this extended curve is not shown here, instead, the sum of the extended curves from the two components' extension is plotted in the heavy red curve.

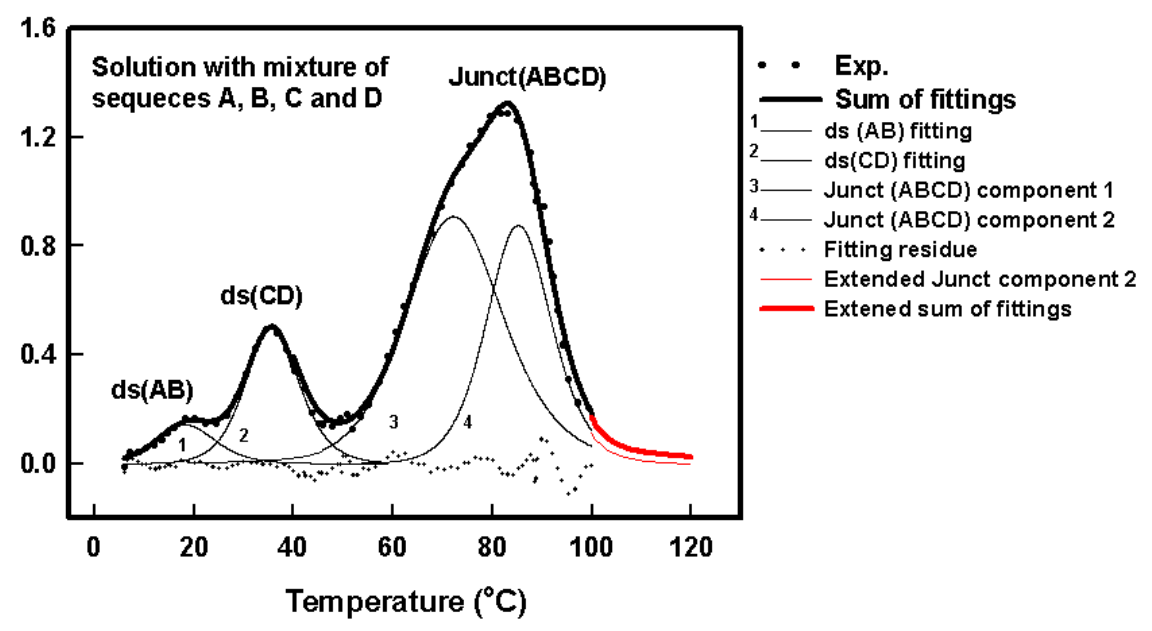

Fig. 4. Deconvolution of the heat capacity scan of ds(AB), ds (CD) and Junct (ABCD) formed from the annealed solutions with $50 \mu \mathrm{M}$ of mixture (ABCD). The solution was prepared in $50 \mathrm{mM}$ sodium cacodylate buffer ( $\mathrm{pH} 7.0)$. The portions of curves in red lines are theoretical extension based on the svmmetrv of fitting combonents. See text for details.

The van't Hoff unfolding thermodynamic qualities obtained from the unfolding modelings of $50 \mu \mathrm{M}$ mixture (ABCD) are summarized in Table 2, together with the results from the $50 \mu \mathrm{M}$ ds( $(\mathrm{AB})$ solution. The junctions' transition enthalpies $\left[\left(\Delta H^{V H}\right)_{1}\right.$ and $\left.\left(\Delta H^{V H}\right)_{2}\right]$ and temperatures $\left[\left(T_{m}\right)_{1}\right.$ and $\left.\left(T_{m}\right)_{2}\right]$ corresponding to the two independent cooperative transitions are both listed in Table 2, along with the sum of enthalpy from the two transitions $\left[\left(\Delta H^{V H}\right)_{\text {sum }}=\left(\Delta H^{V H}\right)_{1}+\left(\Delta H^{V H}\right)_{2}\right]$. The portion of theoretical unfolding enthalpies resulting from the extension are added to the unfolding enthalpies without the extension $\left(\Delta H^{V H}\right)_{2}$ using '+' sign. The adjustment for the first component is smaller and falls within the experimental errors, thus it is not included here.

Table 2. The van't Hoff unfolding enthalpies and temperatures for ds $(A B), \operatorname{ds}(C D)$ and $\operatorname{Junct}(A B C D)$ formed from two different solutions, both were prepared in $50 \mathrm{mM}$ sodium Cacodylate buffer ( $\mathrm{pH} 7.0$ ).

\begin{tabular}{lcccl}
\hline Solutions & $\mathbf{5 0} \boldsymbol{\mu} \mathbf{M}$ of $\mathbf{~ d s}(\mathbf{A B})$ & \multicolumn{3}{c}{ Mixture of $\mathbf{A}, \mathbf{B}, \mathbf{C}$, and D $(\mathbf{5 0} \boldsymbol{\mu} \mathbf{M}$ for each DNA $)$} \\
\hline Conformation & $\mathrm{ds}(\mathrm{AB})$ & $\mathrm{ds}(\mathrm{AB})$ & $\mathrm{ds}(\mathrm{CD})$ & Junct $(\mathrm{ABCD})$ \\
$\begin{array}{l}\boldsymbol{\Delta} \mathbf{H}^{\mathrm{VH}} \\
(\mathbf{K c a l} / \mathbf{m o l})\end{array}$ & 42.1 & 42.6 & 55.1 & $\begin{array}{l}\left(\Delta \mathrm{H}^{\mathrm{VH}}\right)_{1}=36.1 \\
\left(\Delta \mathrm{H}^{\mathrm{VH}}\right)_{2}=58.4+(\sim 3.1)=\sim 61.5 \\
\left(\Delta \mathrm{H}^{\mathrm{VH}}\right)_{\mathrm{sum}}=94.5+(\sim 3.1)=\sim 97.6\end{array}$ \\
& 18.5 & & & \\
& 18.5 & 35.8 & $\begin{array}{l}\left(\mathrm{T}_{\mathrm{m}}\right)_{1}=72.7 \\
\left(\mathrm{~T}_{\mathrm{m}}\right)_{2}=85.4\end{array}$ \\
\hline
\end{tabular}


The ds(AB) formed in the two different solutions are found to have the same van't Hoff unfolding enthalpy $(42 \mathrm{Kcal} / \mathrm{mol})$ and unfolding temperature $\left(18.5^{\circ} \mathrm{C}\right)$, so does the unfolding entropy. This confirms the experimental and analyzed results in this work regarding data collections, data process including sample background collections, and results' modelings. Both of the van't

The two transition temperatures associated with unfolding of Junct(ABCD) are both much higher than that of ds $(\mathrm{AB})$ and $\mathrm{ds}(\mathrm{CD})$, even much higher $\left(13^{\circ} \mathrm{C}+\right)$ than the unfolding temperature found for an immobile junctions formed from 4 of 16-mer DNA sequences $\left(\sim 60^{\circ} \mathrm{C}\right)[13]$. In contrast, the two transition enthalpies related to junctions' unfolding are in different orders compared to the unfolding enthalpies of duplexes: the first transition enthalpy is smaller than that of $\mathrm{ds}(\mathrm{AB})$ and $\mathrm{ds}(\mathrm{CD})$, while the second one is larger than the unfolding enthalpies for both duplexes. The much higher unfolding temperature for junctions show that the molecular interactions that holding four strands of DNA together as junctions are very strong. There are much more heat flow $t$ only leading to faster thermal motions of each junction as an entity (increasing temperature) before the junctions start to unfold.

Why are there two independent cooperative transitions involving in the unfolding of Junct(ABCD)? Is this because there are two conformations of junctions, or the unfolding of a junction $\mathrm{ABCD}$ can start with two sites/units independently? If there are two major conformations for junctions $\mathrm{ABCD}$ in the studies solution, then the two van't Hoff unfolding enthalpies from two modeling components correspond to the unfolding enthalpies of the two forms of junctions $\mathrm{ABCD}$. In this frame, one form of junctions $\mathrm{ABCD}$ has very low unfolding enthalpies $(\sim 36 \mathrm{Kcal} / \mathrm{mol})$, even lower than the unfolding enthalpies of single duplex $\mathrm{AB}$ or $\mathrm{CD}$ that have only 6 complementary Watson-Crick base-pairings. The other form of junctions $\mathrm{ABCD}$ has an unfolding enthalpy only $\sim 6 \mathrm{Kcal} / \mathrm{mol}$ higher than duplexes $\mathrm{CD}$. The low unfolding enthalpies of both forms of $\mathrm{ABCD}$ junctions indicate that the heat needed to unfold the $\mathrm{ABCD}$ junctions is low and the $\mathrm{ABCD}$ junctions are not very thermal stable. This is not consistent with their very high unfolding temperatures. Furthermore, the previous study shows that the unfolding enthalpy of an immobile junction is equal to sum of the unfolding enthalpies of the four constructed junction arms [13], i.e., the sum of the unfolding enthalpies of two duplexes. In this study, the sum of transition enthalpies $\left(\Delta \mathrm{H}^{\mathrm{VH}}\right)_{\text {sum }}=\sim 97.6 \mathrm{Kcal} / \mathrm{mol}$ of the two modeling components is almost equal to the sum of
Hoff unfolding enthalpy and temperature of ds(CD) are larger than those of ds( $\mathrm{AB})$, respectively, although there are the same number (i.e., 6) of complementary WatsonCrick base-pairings in $\operatorname{ds}(\mathrm{AB})$ and $\mathrm{ds}(\mathrm{CD})$. This is possibly because that ds $(C D)$ contains 5 of continuous CG base-pairings (Fig. 2) and CG base pairing is more stable than AT base pairing.

the unfolding enthalpies $(97.7 \mathrm{Kcal} / \mathrm{mol})$ of the two duplexes $\mathrm{AB}$ and $\mathrm{CD}$. Therefore this leads to the other more possible explanation: the unfolding of junctions $A B C D$ can start with two different sites/units independently. The question then is raised: what are the two sites/units? Considering the construction of a junction, the most possible interpretation is: the junctions $\mathrm{ABCD}$ start unfolding either from the junction arms, or from the junction cross-over, or from both of junction arms and cross independently. The molecular interactions at junction cross-over are strong and crucial for the formation of junctions, thus the second component of two-state modeling with higher transition temperature is related to the unfolding of junctions from the junction cross-over. The first component of twostate modeling with lower transition temperature corresponds to the unfolding of junctions $\mathrm{ABCD}$ from the junction arms. The large overlap between the first component and the second component indicates an ABCD junction can unzip from both of junction arms and junction cross-over simultaneously and independently.

3.2 Ionic strength and buffer effects. The annealed solutions of mixture (ABCD) $(50 \mu \mathrm{M}$ for each DNA sequence) were studies not only in a buffer of $50 \mathrm{mM}$ sodium cacodyalte (7.0) but also in a $50 \mathrm{mM}$ sodium phosphate buffer ( $\mathrm{pH}$ 7.0). Fig. 5 shows the experimental excess heat capacity scan of the mixture in sodium phosphate in dotted curve. It is obvious that there are still a certain amount of $\mathrm{ds}(\mathrm{AB})$ and $\mathrm{ds}(\mathrm{CD})$ beside the relative large amount of Junct (ABCD). The experimental data is modeled with two-state functions. The lighter solid black curves from left to right (labeled as 1,2,3, and 4) correspond to the modelings for ds $(A B), d s(C D)$ and Junct (ABCD), respectively. The sum of these modelings (heavy solid black curve) gives a very good approximate to the experimental data. The red curve is the extension of Junct(ABCD) modeling component 2 based on the symmetry of this component, which is also the extended part for the sum of all modelings. The thermodynamic parameters derived from the modelings are listed in Table 3.

Compared with the unfolding thermodynamic parameters in $50 \mathrm{mM}$ sodium cacodylate buffer in Table 2, 
in $50 \mathrm{mM}$ sodium phosphate buffer, both ds $(\mathrm{AB})$ and $\mathrm{ds}(\mathrm{CD})$ exhibit a few degree Celsius higher in unfolding temperature, while $\sim 10 \mathrm{Kcal} / \mathrm{mol}$ lower in unfolding enthalpy. The controversy changing direction in unfolding temperature and enthalpy of both duplexes results in a decrease in the unfolding entropy of the duplexes. This indicates that the microstates (entropy) of duplexes are larger in a $50 \mathrm{mM}$ sodium phosphate buffer than in a $50 \mathrm{mM}$ sodium cacodylate buffer. The dramatic difference between the DNA mixed solutions in the two different buffers lies in the transition enthalpies and temperature related to the unfolding of Junct(ABCD).In sodium phosphate buffer, the transition temperatures of the two fitting components are more than $20^{\circ} \mathrm{C}$ higher. The transition enthalpy of the first component is nearly 4 times larger. The transition enthalpy of the second component cannot be accurately determined since the temperature limit of the instrument and the temperature limit for buffer's stability. As shown in the red curve in Fig.5, the unfolding of Junct $(\mathrm{ABCD})$ is extended theoretically to higher temperature using the symmetry of the second fitting component. The transition enthalpy resulting from this extension shown in italic font in Table $\mathbf{3}$ is added to the transition enthalpy of the non-completed second fitting component to give an estimated unfolding enthalpy of the second transition. This estimated transition enthalpy is almost twice larger in sodium phosphate buffer than in sodium cacodylate buffer.

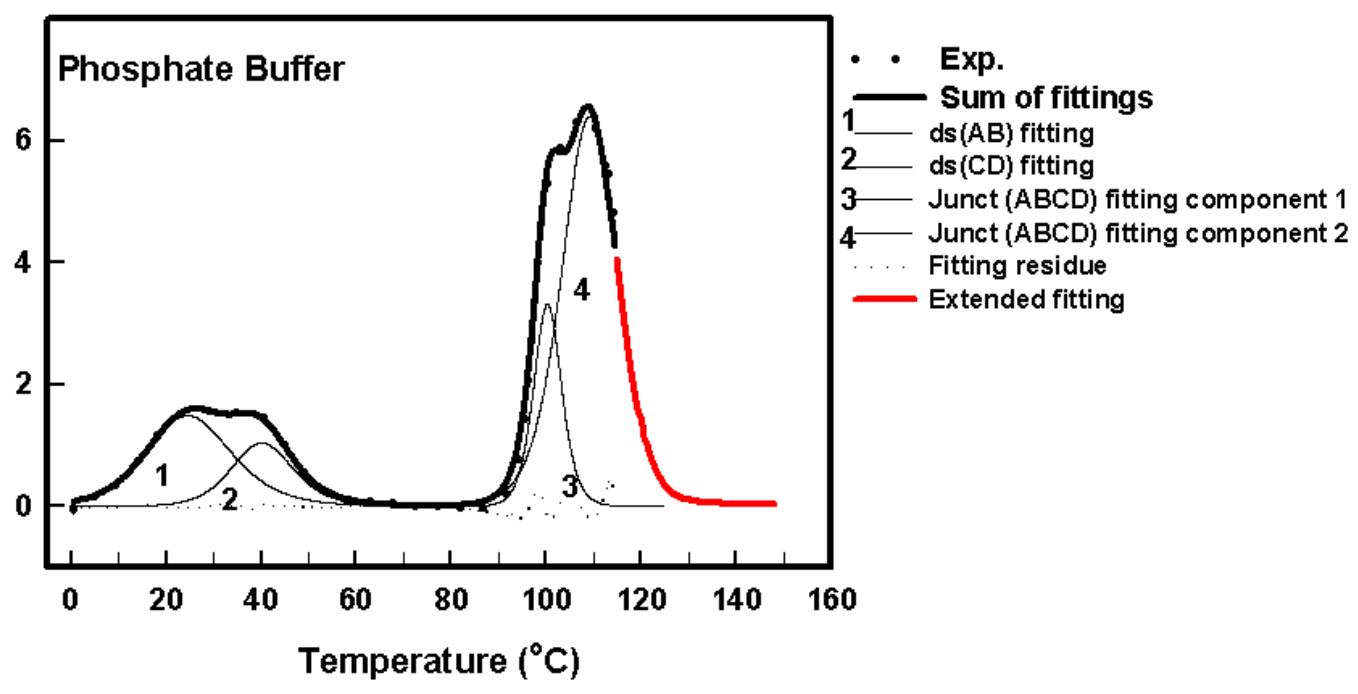

Fig. 5. Deconvolution of the heat capacity scan of ds(AB), ds (CD) and Junct (ABCD) formed from the annealed mixture $(\mathrm{ABCD})$ solution $(50 \mu \mathrm{M}$ of sequences $\mathrm{A}, \mathrm{B}, \mathrm{C}$, and $\mathrm{D}$, respectively). The solution was prepared in $50 \mathrm{mM}$ sodium phosphate buffer ( $\mathrm{pH} 7.0)$. The portions of curve in red lines are theoretical extension based on the symmetry of fitting components.

Table 3. The van't Hoff unfolding enthalpies and temperatures for ds(AB), ds(CD) and Junct(ABCD) formed from the annealed solution with four DNA sequences A, B, C and D $(50 \mu \mathrm{M}$ of each DNA). The solutions were prepared in $50 \mathrm{mM}$ sodium phosphate buffer ( $\mathrm{pH} 7.0)$.

\begin{tabular}{lcll}
\hline Conformation & ds(AB) & ds(CD) & Junct $(\mathbf{A B C D})$ \\
\hline$\Delta \mathbf{H}^{\mathrm{VH}}(\mathbf{K c a l} / \mathbf{m o l})$ & 29.5 & 44.9 & $\left(\Delta \mathrm{H}^{\mathrm{VH}}\right)_{1}=142.1$ \\
& & & $\left(\Delta \mathrm{H}^{\mathrm{VH}}\right)_{2}=72.4+(\sim 41.8)=$ \\
& & $\sim 114$ \\
$\mathbf{T}_{\mathbf{m}}{ }^{\mathrm{VH}}\left({ }^{\circ} \mathbf{C}\right)$ & 25.0 & 40.4 & $\left(\mathrm{~T}_{\mathrm{m}}\right)_{1}=100.3$ \\
& & & $\left(\mathrm{~T}_{\mathrm{m}}\right)_{2}=109.3$ \\
\hline
\end{tabular}


Are the two modeling components of Junct (ABCD) formed in sodium phosphate buffer still resulted from the two different cooperative sites/units during the unfolding of a single junction? The much larger transition enthalpies from the two modeling components show that this is hardly possible. Instead, it is more possible that the two components are corresponding to two different conformations of $\mathrm{ABCD}$ junctions. Although the $50 \mathrm{mM}$ sodium cacodylate buffer and 50 $\mathrm{mM}$ sodium phosphate buffer have the same buffer concentration and contain the same type of cation $\mathrm{Na}^{+}$, the ionic strength of $50 \mathrm{mM}$ sodium phosphate is 113 $\mathrm{mM}$, more than twice larger than the ionic strength of 50 $\mathrm{mM}$ sodium cacodylate $(50 \mathrm{mM})$. Furthermore, the concentration of $\mathrm{Na}^{+}$is $80 \mathrm{mM}$ in sodium phosphate, also much larger than that in sodium cacodylate. Many studies show that the DNA Holliday junctions intend to have an open-X conformation in solution with low ionic strength with junctions' four arms extending far away from each other to reduce the electrostatic repulsion between the negatively-charged phosphate groups on backbones $[1,14]$. In presence of divalent metal ions or with higher ionic strength, the DNA Holliday junction can be folded from open-X conformation into stacked-X conformation [14, 15, 16, 17]. In this work, in a buffer of $50 \mathrm{mM}$ sodium cacodylate with low ionic strength, Junct(ABCD) are expect to exist in open-X form, while in a buffer of $50 \mathrm{mM}$ sodium phosphate buffer with much higher ionic strength and more concentrated $\mathrm{Na}^{+}$ to neutralize the phosphate groups on backbones, open$X$ Junct(ABCD) can be folded into stacked-X form. In such a situation, the transition enthalpies from the two modeling components are corresponding to the unfolding enthalpies of two forms of $\mathrm{ABCD}$ junctions, different from the cases found in $50 \mathrm{mM}$ sodium cacodylate buffer which correspond to two cooperative sites/units of a junction.

What are two forms of Junct(ABCD) formed in the DNA solution in phosphate buffer? Firstly, it is helpful to compare the transition enthalpies from the unfolding of junctions $A B C D$ in different buffers. In sodium cacodylate buffer, the junctions $\mathrm{ABCD}$ are in open-X form and the unfolding of an open-X Junct(ABCD) proceeds with two different sites simultaneously and independently with corresponding transition parameters $\left(\Delta H^{V H}\right)_{S I}=36 \mathrm{Kcal} / \mathrm{mol},\left(T_{m}\right)_{S I}=73^{\circ} \mathrm{C}$ and $\left(\Delta H^{V H}\right)_{S 2}=62$ $\mathrm{Kcal} / \mathrm{mol}, \quad\left(T_{m}\right) S_{2}=85^{\circ} \mathrm{C}$ for each unfolding sites, respectively. The total unfolding enthalpy of open-X Junct $(A B C D)$ is $\left(\Delta \mathrm{H}^{\mathrm{VH}}\right)_{\text {sum }}=36+62=98 \mathrm{Kcal} / \mathrm{mol}$. In sodium phosphate buffer, open-X Junct(ABCD) can be folded into stacked-X form. The modeling of the unfolding profile of junctions $(\mathrm{ABCD})$ indicate that there are two forms of junctions existed and the unfolding parameters for the two junction forms are $\left(\Delta H^{V H}\right)_{J I}=142 \mathrm{Kcal} / \mathrm{mol},\left(T_{m}\right)_{J I}=100^{\circ} \mathrm{C}$ and $\left(\Delta H^{V H}\right)_{J 2}=$ $114 \mathrm{Kcal} / \mathrm{mol},\left(T_{m}\right)_{J 2}=109^{\circ} \mathrm{C}$, respectively. One should note that the transition enthalpy from the second fitting component has large theoretic portion based on the symmetric extension of the modeling peak. Any certain inaccurate prediction from this extension may result in some changes in the modeling results of the two components. These changes, however, will not be bigger enough to alter difference magnitude of either transition enthalpies or temperatures for the Junct(ABCD) formed in the two different buffers. The unfolding enthalpies of both forms of junctions in sodium phosphate are larger than the unfolding enthalpy of open-X junctions in sodium cacodylate. The open-X form should be destabilized in solutions with higher ionic strength and more cations, and thus open-X junctions $(\mathrm{ABCD})$ in sodium phosphate buffer, if there are any, should have lower unfolding enthalpy and temperature. Is this indicative of that all open-X junctions are folded into stacked-X form(s)? Not necessary, because possibly most of $\mathrm{Na}^{+}$ions involve in the folding of stacked-X and interact with stacked-X junctions, resulting in an local environment with much lower ionic strength and diluted $\mathrm{Na}^{+}$than expected for open-X junctions. The two forms of $\mathrm{ABCD}$ junctions, therefore, can be open-X and stacked-X. However there is still another possibility that open-X ABCD junctions are completely folded into parallel stacked-X and antiparallel stacked-X forms, but previous study [1] suggested that the parallel stacked-X conformation is not possible for an asymmetric junction that Junc(ABCD) is based on. With all the known knowledge, therefore the two most possibly forms of $A B C D$ junctions formed in sodium phosphate buffer are open-X and antiparallel stacked-X.

DNA Holliday junctions' switch induced by ionic strength between open-X and stacked-X forms has been studied by Mount, et al. [18] using fluorescence resonance energy transfer. It was found that $\left[\mathrm{Na}^{+}\right]=$ $\sim 0.16 \mathrm{M}$ is needed to have half of open-X junctions convert to stacked-X junctions in a solution of $1 \mu \mathrm{M}$ studied open-X Holliday junction in Tris buffer $\left(\left[\mathrm{Na}^{+}\right] /[\mathrm{Junct}]=1.6 \times 10^{-7}\right)$. In this work, the studies $\mathrm{ABCD}$ junction is formed from $50 \mu \mathrm{M}$ mixture of four sequences. Since there are some duplexes $(\operatorname{ds}(\mathrm{AB})$ and $\mathrm{ds}(\mathrm{CD})$ ) are presented, the concentration of $\mathrm{ABCD}$ junctions is not $50 \mu \mathrm{M}$, but should not be very small and should be much larger than $1 \mu \mathrm{M}$ according to the heat capacity scans. However in the studied system where significant amount of junction switches are observed, 
the $\left[\mathrm{Na}^{+}\right]$is equal to $82 \mathrm{mM}(0.082 \mathrm{M})\left[\left(\left[\mathrm{Na}^{+}\right] /[\mathrm{Junct}]\right.\right.$ $=1.6 \times 10^{-9}$ if $\left.[\mathrm{Junct}]=50 \mu \mathrm{M}\right)$. The profound difference in the required $\mathrm{Na}^{+}$concentration for junction switch may be caused by different DNA Holliday junctions and different buffers. It is surprised to observe junction conformation switch in such low ionic strength in this work, the difference between $50 \mathrm{mM}$ sodium cacodylate buffer and sodium phosphate buffer lies not only on their ionic strength and $\mathrm{Na}^{+}$concentration, but also on the anions. It is unknown whether phosphate ions from the buffer may facilitate the junction switch.

3.3 Pressure effects on junction unfolding. During DSC scans of the annealed DNA solutions in two different buffers discussed above, the DSC sample chamber was pressurized to $3 \mathrm{~atm}$, a recommended standard pressure for TA nano-DSC in order to prevent the solutions from boiling at high temperature. TA
nano-DSC offers a range of operating pressure up to 6 atm. In order to evaluate hydration effects on the unfolding of DNA junctions, the DSC scans were recorded under $5 \mathrm{~atm}$ chamber pressure for the annealed solutions with $50 \mu \mathrm{M}$ mixture (ABCD) in $50 \mathrm{mM}$ sodium cacodylate buffer. The experimental excess heat capacity scan is shown in dotted curve in Fig. 6. The formation of both duplexes ds(AB) and $\mathrm{ds}(\mathrm{CD})$ and junctions $(\mathrm{ABCD})$ are all detectable as in the above studies. The unfolding of all these DNA conformations, i.e., ds(AB), ds(CD) and Junct(ABCD), are modeled with two-state functions, as shown in light solid black curves labeled as 1,2,3 and 4, respectively. The sum of the four modeling curves shown in heavy solid curve is in good agreement with the experimental data. Again, the red curves are the extension to higher temperature based on the symmetry of the modeling curves.

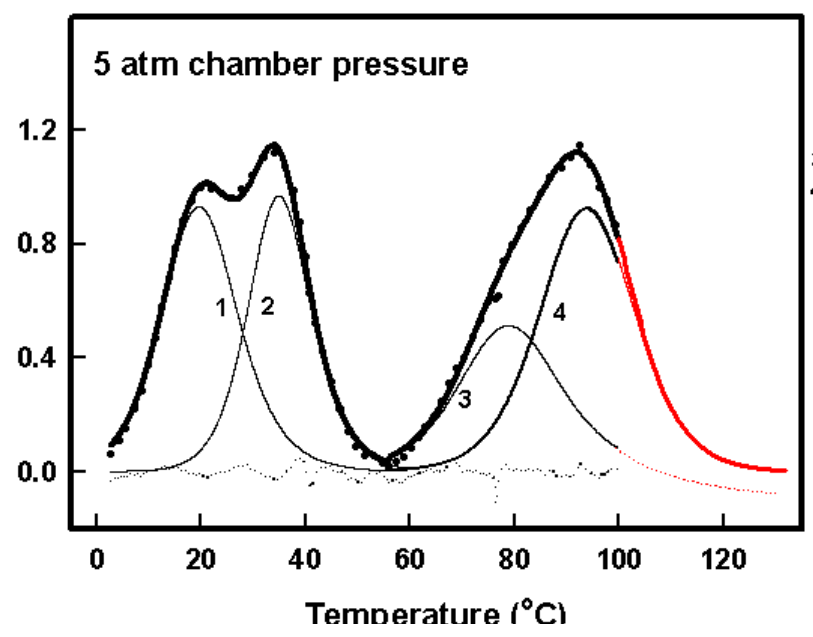

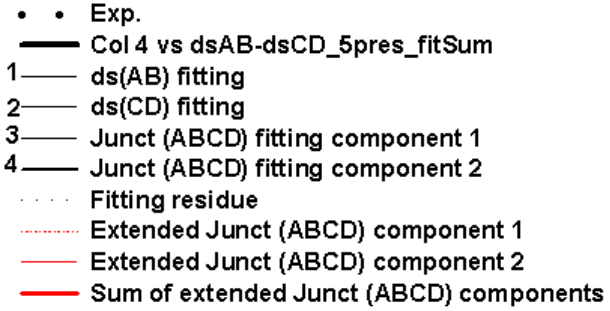

Fig. 6. Deconvolution of the heat capacity scan of ds(AB), ds (CD) and Junct (ABCD) under $5 \mathrm{~atm}$ chamber pressure. The DNA complexes were formed from the annealed solutions with $50 \mu \mathrm{M}$ of sequences $\mathrm{A}, \mathrm{B}, \mathrm{C}$, and $\mathrm{D}$, respectively, in $50 \mathrm{mM}$ sodium cacodylate buffer ( $\mathrm{pH} 7.0)$ at atmospheric pressure. The portions of curves in red lines are theoretical extension based on the symmetry of modelig components.
Table 4 summarizes the van't Hoff enthalpies and temperatures obtained from two-state modeling of $\mathrm{ds}(\mathrm{AB}), \mathrm{ds}(\mathrm{CD})$ and Junct(ABCD) to the experimental heat capacity scans under $5 \mathrm{~atm}$ chamber pressure. Interestedly, by comparison of Table 2 and Table 4, it is found that increasing in chamber pressure results in opposite effects on the unfolding qualities on duplexes and junctions. For duplexes $\mathrm{ds}(\mathrm{AB})$ and $\mathrm{ds}(\mathrm{CD})$, the increasing in chamber pressure does not change the unfolding temperature but decreases the unfolding enthalpies by $7 \sim 8 \mathrm{Kcal} / \mathrm{mol}$. For $\mathrm{ABCD}$ junctions, the increasing in chamber pressure does not change the transition enthalpies of both components but increases the unfolding temperatures of each component by $7^{\circ} \mathrm{C}$ and $9^{\circ} \mathrm{C}$, respectively.

According to Clapeyron equation [19, 20], $\frac{d T_{m}}{d P}=T_{m} \frac{\Delta V}{\Delta H}, \quad$ the temperature change $\Delta T_{m}$, upon a pressure change $\Delta p$, can be used to calculate the volume change $\Delta V$ during unfolding, provided the known unfolding enthalpy $\Delta H$ is known. The temperature changes for the two duplexes $\operatorname{ds}(\mathrm{AB})$ and 
$\mathrm{ds}(\mathrm{CD})$ is negligible within the experimental errors, thus the molar volumes of the two duplexes stay nearly unchanged during heat-induced unfolding. This is different from the prediction that a polymeric duplex is destabilized by pressure $(\Delta \mathrm{V}<0)$ if its melting temperature $\mathrm{T}_{\mathrm{m}}<\sim 50^{\circ} \mathrm{C}$ and stabilized by pressure $(\Delta \mathrm{V}>0)$ if the $\mathrm{T}_{\mathrm{m}}>\sim 50^{\circ} \mathrm{C}$, based on the pressuretemperature phase diagram for polymeric nucleic acids published by Dubins et al. [21]. However a study by showed that some oligomeric nucleic acid duplexes in fact are stabilized by pressure (positive $\Delta \mathrm{V}$ ) even though in some cases that the transition temperatures are much lower than $50^{\circ} \mathrm{C}$ [22], because Oligomeric nucleic acid duplexes have much shorter sequences and their stabilities are affected due to the greater relative proportion of terminal bases (end-fraying).

Table 4. The van't Hoff unfolding enthalpies and temperatures derived for ds(AB), ds(CD) and Junct(ABCD) from the excess heat capacity scan recorded under 5 atm chamber pressure. The different DNA conformations are

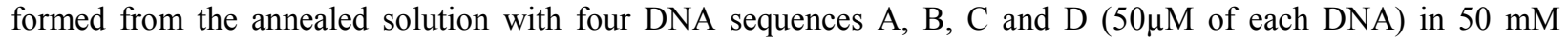
sodium cacodylate buffer ( $\mathrm{pH} 7.0)$ at atmosphere pressure.

\begin{tabular}{lcll}
\hline Conformation & $\mathbf{d s}(\mathbf{A B})$ & $\mathbf{d s}(\mathbf{C D})$ & Junct $(\mathbf{A B C D})$ \\
\hline$\Delta \mathbf{H}^{\mathbf{V H}}(\mathbf{K c a l} / \mathbf{m o l})$ & 35.1 & 47.2 & $\left(\Delta \mathrm{H}^{\mathrm{VH}}\right)_{1}=38.1$ \\
& & & $\left(\Delta \mathrm{H}^{\mathrm{VH}}\right)_{2}=72.4+(\sim 18.2)=\sim 62.1$ \\
& 19.9 & 35.1 & $\left(\mathrm{~T}_{\mathrm{m}}\right)_{1}=79.3$ \\
$\mathbf{T}_{\mathbf{m}}{ }^{\mathrm{VH}}\left({ }^{\circ} \mathbf{C}\right)$ & & & $\left(\mathrm{T}_{\mathrm{m}}\right)_{2}=94.2$ \\
\hline
\end{tabular}

For Junct(ABCD), the molar volume change of the first transition $\left(\Delta V_{l}\right)$ of heat-induced unfolding of Junct (ABCD) is calculated to be $\Delta V_{l}=+14 \mathrm{~cm}^{3} / \mathrm{mol}$, and the molar volume change of the second transition is $\Delta V_{2}=$ $+31 \mathrm{~cm}^{3} / \mathrm{mol}$. The molar volume change $(\Delta \mathrm{V})$ can be defined as the difference between the molar volume of the products and that of the reactants. The positive sign of volume changes indicates that the molar volume of 4 single strands (usually in coil form) is larger than the molar volume of the (ABCD) junction. Compared with single strands, there are no formations of stronger interactions between junctions and water solvent, in other words, no more extensive hydration formed for the studied junctions. As already discussed above, the first transition during $\mathrm{ABCD}$ junction unfolding corresponds to the unzip of junction arm(s), and the second transition corresponds to unzip of junction cross-over. The molar volume change associated with the second transition is more than twice more positive than that associated with the first transition. This is not surprised since the junction cross-over is less water accessible and thus less extensive hydrated.

\section{Conclusion}

An asymmetric junction of known crystal structure, constructed from for unique DNA sequences A, B, C and $\mathrm{D}$, has been selected for the thermodynamic study using nano-DSC. Fig. 7 summarizes the DSC thermal scans of all the studied solutions of $A B C D$ mixture. It is found that the DNA in the ABCD mixture solutions can form duplexes ds $(A B)$ and $d s(C D)$, and junctions $A B C D$. These thermal scans are modeled with two-state functions to obtain the van't Hoff thermodynamic qualities associated with the unfolding of duplexes (AB), (CD) and junctions (ABCD).

According to the determined thermodynamic qualities, it has been concluded that (ABCD) junctions in $50 \mathrm{mM}$ sodium cacodylate (curve1) exist in an open-X form and the unfolding of an open-X (ABCD) junction can proceeds from two different sites simultaneously and independently. Because junctions in open- $X$ form can undergo migrate along the DNA sequence but junctions in antiparallel stacked-X cannot unless they adopt open$\mathrm{X}$ form, this information can be helpful in understanding the mechanism of branch migration.

Furthermore, in $50 \mathrm{mM}$ sodium phosphate buffer (curve2) with higher ionic strength and more concentrated $\mathrm{Na}+$, it was found that some $\mathrm{ABCD}$ junctions in open-X form are folded into stacked-X form. The unfolding enthalpies and temperatures were detected for junction in both (ABCD) junction conformations existed in the same solution. The difference in their unfolding thermal parameters will help understand the energetic barriers for junction conformational switch and thus protein bindings which require junction's adopting of open-X conformation. 


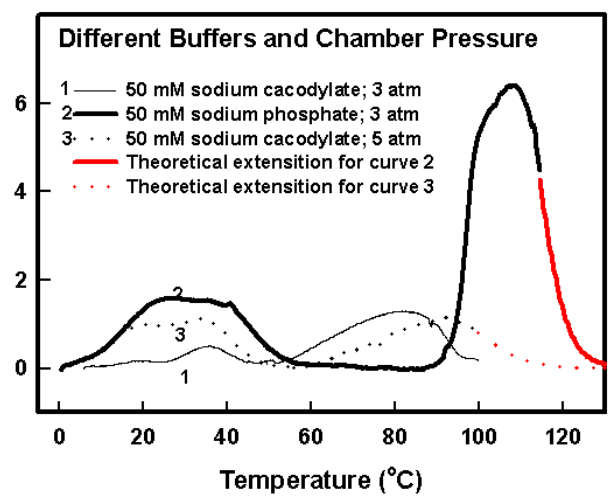

Fig. 7. Excess heat capacities of ds(AB), ds (CD) and Junct $(\mathrm{ABCD})$ as temperature. The DNA complexes were formed from the annealed solutions with $50 \mu \mathrm{M}$ of sequences $\mathrm{A}, \mathrm{B}, \mathrm{C}$, and $\mathrm{D}$, respectively, in $50 \mathrm{mM}$ sodium cacodylate buffer (pH7.0) (Curve 1), or $50 \mathrm{mM}$ sodium phosphate (pH 7.0) (Curve 2), respectively. The DSC thermal scans were recorded under different chamber pressure: $3 \mathrm{~atm}$ (curve 1 and 2) and 5 atm (curve 3) The portions of curves in red lines are theoretical extension based on the symmetry of modeling components.
The molar volume change of heat-induced unfolding of open-X Junct (ABCD) is determined by applying different chamber pressure on the same studied DNA solution (comparison of curves 3 and 1). The positive sign of volume changes indicates that the hydration of open-X junctions is not very strong. The hydration of junction arms is stronger than that of junction cross-over, as indicated by the more positive volume changes at cross-over compared to junction arms. Hydration of a

\section{Acknowledgment}

This work was supported by funding from industry.

\section{Reference}

[1]. Duckett, D.R.; Murchie, A.I.H.; Diekmann S.; von Kitzing. E.; Kemper, B.; Lilley, D.M.J. The structure of the Holliday junction, and its resolution. Cell 1988, 55, 79-89.

[2] Khuu, P.; Ho, P. S. A rare nucleotide base tautomer in the structure of an asymmetric DNA junction. Biochemistry. 2009, 48(33), 7824-7832.

[3] Hays, F.A.; Teegarden, A.; Jones, Z.J.; Harms, M.; Raup, D.; Watson, .J; Cavaliere, E.; Ho, P.S. How sequence defines structure: a crystallographic map of DNA structure and conformation. Proc. Natl. Acad. Sci.2005,102,7157-7162.

[4] Thorpe, J.H.; Gale, B.C.; Teixeira, S.C.; Cardin, C.J.; Conformational and Hydration Effects of Site-selective Sodium, Calcium and Strontium Ion Binding to the DNA Holliday Junction Structure d

(TCGGTACCGA). J. Mol. Biol 2003, 327, 97-109.

[5] Eichman, B.F.; Vargason, J.M.; Mooers, B.H.M.; Ho, P.S. The Holliday junction in an inverted repeat

sequence: sequence effects on the structure of four-way junctions. Proc. Natl. Acad. Sci. USA

2000, 97, 3971-3976.

[6] Ortiz-Lombardía, M.; González, A.; Eritja, R.; Aymamí, J.; Azorín, F.; Coll, M. Crystal structure of a DNA

http://ccaasmag.org/BIO junction will affect its interactions with other molecules such as ions and proteins. It is expected to compare the results in this work from two thermal scans under different DSC chamber pressure to the junction unfolding study using pressure perturbation calorimetry (PPC). However, little studies have been conducted on the pressure-perturbed unfolding of Holliday junctions using PPC.

Holliday junction. Nat. Struct. Biol 1999, 6, 913-917.

[7] Plum, G. E.; Breslauer, K. J. Calorimetry of proteins and nucleic acids. Current Opinion in Structural Biology, 1995, 5(5), 682-690.

[8] Mikulecky, P. J.; Feig, A.L. Heat capacity changes associated with nucleic acid folding. Biopolymers. 2006, 82(1), 38-58.

[9] Mason, J. T. Investigation of phase transitions in bilayer membranes. Methods Enzymol. 1998, 295, 468-494.

[10] Privalov, P. L.; Dragan, A. I. Microcalorimetry of biological macromolecules. Biophy.Chem.2007, 126, 16-24.

[11] Wber, P. C.; Salemme, F. R. Applications of calorimetric methods to drug discovery and the study of protein interactions. Current Opinion in Structural Biology. 2003, 13(1), 115-121.

[12] Privalov, P. L.; Potekhin, S. A. Scanning microcalorimetry in studying temperature-induced changes in proteins. Methods in enzymology. 1986, 131, 4-51.

[13] Marky, L. A.; Kallenbach, N. R.; McDonough, K. A.; SeeMan, N.C.; Breslauer, K. J. The melting behavior of a DNA Junction Structure: A calorimetric and spectroscopic study. Biopolymers, 1987, 26, 1621-1634.

[14] Clegg,R.M.; Murchie, A.I.H.; Lilley, D.M.J. The solution structure of the four-way DNA junction at low-salt conditions: a fluorescence resonance energy transfer analysis. Biophys. J., 1994, 66, 99-109.

[15] Churchill, M.E.; Tullius, T.D.; Hallenbach, N.R.; Seeman, N.C.A. Holliday recombination intermediate is twofold symmetric. Proc. Natl Acad. Sci. USA, 1988, 85, 4653-4656. 
[16] Murchie, A.I.H.; Clegg, R.M.; von Kitzing, E.; Duckett, D.R.; Diekmann, S.; Lilley, D.M.J. Flurorescence energy transfer shows that the four-way DNA junction is a right-handed cross of antiparallel molecules. Nature, 1989, 341, 763-766.

[17]Cooper, J.P.; Hagerman, P.J. Geometry of a branched DNA structure in solution. Proc. Natl Acad. Sci. USA, 1989, 86, 73367340 .

[18] Mount, A.R.; Mountford, C.P.; Evans, S.A.G.; Su, T.-J.; Buck, A.H.; Dickinson, P.; Campbell, C.J.; Keane, L.M.; Terry, J.G.; Beattie, J.S.; Walton, A.J.; Ghazal, P.; Crain, J. Biophysical Chemistry, 2006, 124, 214-221.
[19] Anthony, F. H.; Biltonen, R. L. ; Freire, E. Modification of a vibrating tube density meter for precise temperature scanning. Anal. Biochem. 1981, 116:161-167.

[20] Landwehr, A.; Winter, R. High-pressure differential thermal analysis of lamellar to lamellar and lamellar to non-lamellar lipid phase transitions. Ber. Bunsenges. Phys. Chem. 1994, 98, 214 218.

[21] Dubins, D. N.; Lee, A.; et al. "On the stability of double stranded nucleic acids." J. Am. Chem. Soc. 2001, 123(38): 9254-9259.

[22] Macgregor, R. B., Jr. Chain length and oligonucleotide stability at high pressure. Biopolymers, 1996, 38(3), 321-327. 